\title{
Toluene Biodegradation using a Lab-Scale Biofilter Inoculated with Pseudomonas Putida PTCC 1694
}

\section{CURRENT STATUS: UNDER REVIEW}

AMB Express Springer

\section{Roohollah Ghasemi}

Tehran University of Medical Sciences

farideh golbabaei

Tehran University of Medical Sciences

• ghasemi63@hotmail.comCorresponding Author

Mohammad Reza Pourmand

Tehran University of Medical Sciences

Sasan Rezaei

Tehran University of Medical Sciences

Mohammad Javad Jafari

Shaheed Beheshti University of Medical Sciences

Ramin Nabizadeh

Tehran University of Medical Sciences

Ensieh Masoorian

Tehran University of Medical Sciences

DOI:

$10.21203 / \mathrm{rs} .2 .24116 / \mathrm{v} 1$

\section{SUBJECT AREAS}

General Biochemistry

KEYWORDS

Toluene, Pseudomonas putida, Biodegradation, Elimination Capacity, Removal Efficiency, Pressure Drop 


\section{Abstract}

Biomanipulation is reliable method for treating volatile organic compounds (VOCs) in polluted air. The performances of two biofilters in the removal of toluene vapors from air stream were compared. Two identical biofilters designed in parallel configuration were operated in lab-scale for 20 days; one of them was filled by sterilized media (compost and wood charcoal 2:1 v/v) and another was filled by the same media inoculated with Pseudomonas putida PTCC 1694, as a native strain. Moreover, batch tests were performed to determine the biodegradation rate of toluene. The results showed that, in comparison with the sterilized BF ( $89 \%$ vs $58 \%$ ), the inoculated BF could effectively eliminate toluene from air stream. The pressure drop across the inoculated BF and the sterilized BF were $0.66 \pm 0.28$ and $0.47 \pm 0.27 \mathrm{~mm}$ water respectively. The batch test results showed that loss of toluene in the control bottles was greater than the blanks. Based on the experimental results, inoculated BFs can effectively treat toluene vapors from gaseous streams.

\section{Full-text}

Due to technical limitations, full-text HTML conversion of this manuscript could not be completed.

However, the manuscript can be downloaded and accessed as a PDF.

\section{Tables}

Table 1.

\begin{tabular}{|l|l|l|}
\hline Item & Compost & Wood Charcoal \\
\hline Particle mean diameter (mm) & $2-4$ & $15 \pm 0.5$ \\
\hline Void fraction (\%) & 55 & 50 \\
\hline Wet bulk density (Kg/L) & 0.628 & 0.8 \\
\hline Dry bulk density (Kg/L) & 0.302 & 0.44 \\
\hline Moisture Content (\%) & $48-70$ & $43-67$ \\
\hline Moisture-Holding Capacity (\%) & 72 & 78 \\
\hline
\end{tabular}

Table 2. 


\begin{tabular}{|c|c|c|c|}
\hline \multicolumn{2}{|c|}{ Trace element } & \multicolumn{2}{c|}{ Macro element } \\
\hline compounds & Concentration $\left(\mathrm{mgl}^{-1}\right)$ & Compounds & Concentration $\left(\mathrm{gl}^{-1}\right)$ \\
\hline $\left.\begin{array}{c}\mathrm{CaCl}_{2} \cdot 2 \mathrm{H}_{2} \mathrm{O} \\
\text { EDTA Na}\end{array} \mathrm{H}_{2} \mathrm{O}\right)_{2}$ & 26 & $\mathrm{KH}_{2} \mathrm{PO}_{4}$ & 1 \\
\cline { 2 - 4 } & 5.5 & $\mathrm{~K}_{2} \mathrm{HPO}_{4}$ & 0.2 \\
\hline $\mathrm{FeCl}_{3} \cdot 4 \mathrm{H}_{2} \mathrm{O}$ & 1.3 & $\mathrm{NaCl}$ & 1 \\
\hline $\mathrm{CoCl}_{2} \cdot 6 \mathrm{H}_{2} \mathrm{O}$ & 0.12 & $\mathrm{KNO}_{3}$ & 1 \\
\hline $\mathrm{MnCl}_{2} \cdot 2 \mathrm{H}_{2} \mathrm{O}$ & 100 & & \\
\hline $\mathrm{ZnCl}_{2}$ & 0.07 & & \\
\hline $\mathrm{H}_{3} \mathrm{BO}_{3}$ & 0.06 & & \\
\hline $\mathrm{NiCl}_{2} \cdot 6 \mathrm{H}_{2} \mathrm{O}$ & 0.025 & & \\
\hline $\mathrm{NaMoO}_{4} \cdot 2 \mathrm{H}_{2} \mathrm{O}$ & 0.025 & & \\
\hline $\mathrm{CuCl}_{2} \cdot 2 \mathrm{H}_{2} \mathrm{O}$ & 0.015 & & \\
\hline
\end{tabular}

Table 3.

\begin{tabular}{|l|l|l|l|l|l|}
\hline Part & Day & $\mathrm{C}_{\text {in }}\left(\mathrm{mg} / \mathrm{m}^{3}\right)$ & $\mathrm{LR}\left(\mathrm{g} / \mathrm{m}^{3} \cdot \mathrm{h}\right)$ & $\mathrm{EC}\left(\mathrm{g} / \mathrm{m}^{3} \cdot \mathrm{h}\right)$ & $\mathrm{RE}(\%)$ \\
\hline Sterilized BF (A) & $1-20$ & $134.5 \pm 26.2$ & $11.56 \pm 2.26$ & $6.22 \pm 1.45$ & $52.96 \pm 4.12$ \\
\hline Inoculated BF (B) & $1-20$ & $134.5 \pm 26.2$ & $11.65 \pm 2.26$ & $9.2 \pm 2.65$ & $77.21 \pm 13.55$ \\
\hline
\end{tabular}

Figures

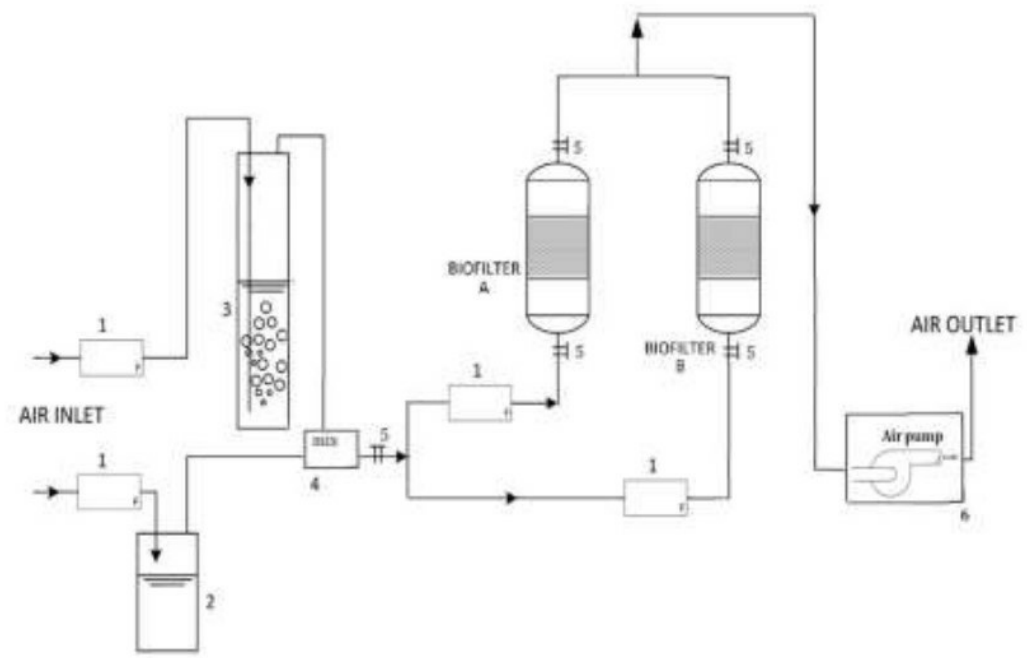

Figure 1

Experimental set-up of the biofilter system: 1. Gas flow controllers, 2. Toluene evaporation chamber, 3. Humidifying column, 4. Mix chamber, 5. Gas sampling ports, 6. Air pump. 


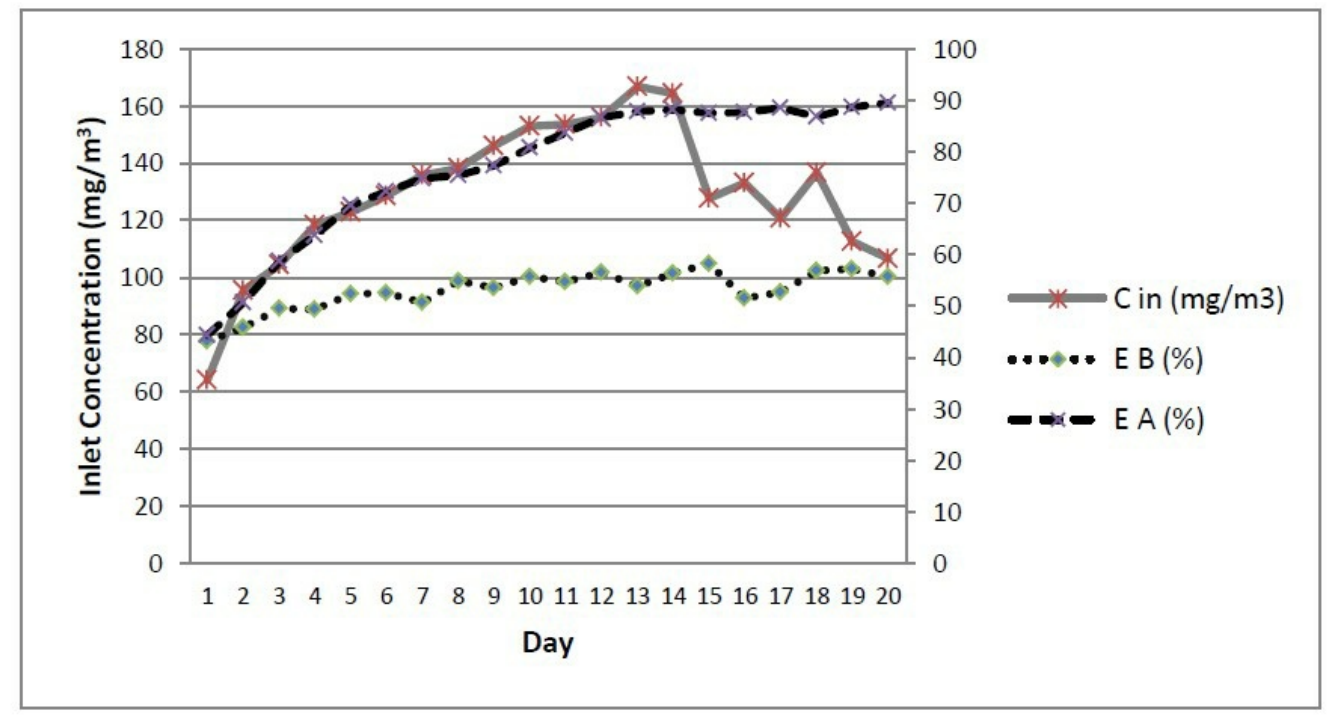

Figure 2

Influence of inlet toluene concentration on the removal efficiency of the BFs (BF-A, BF-B) as a function of time 


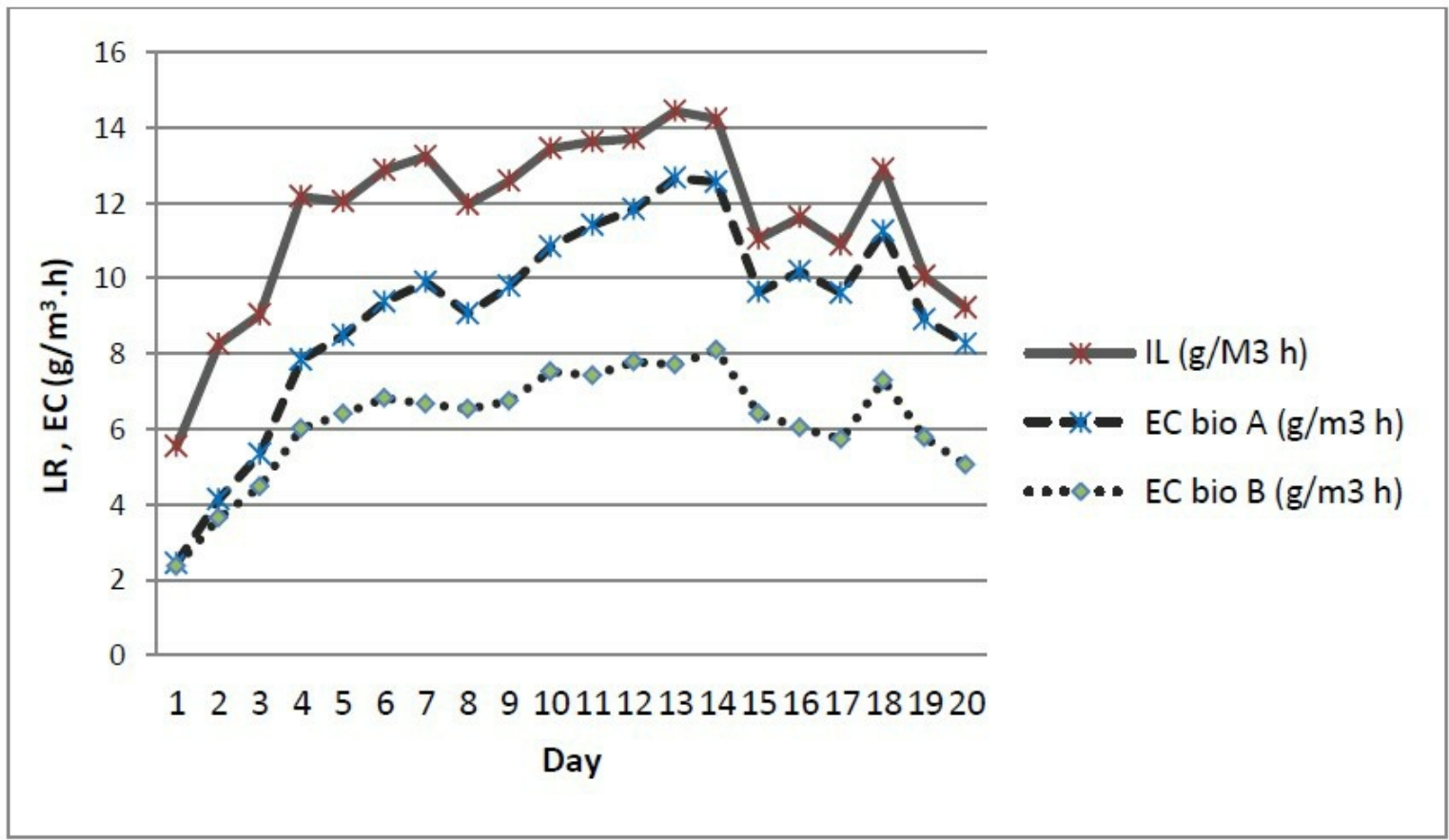

Figure 3

Influence of inlet toluene loading on the elimination capacity of the BFs (BF-A, BF-B) as a function of time 


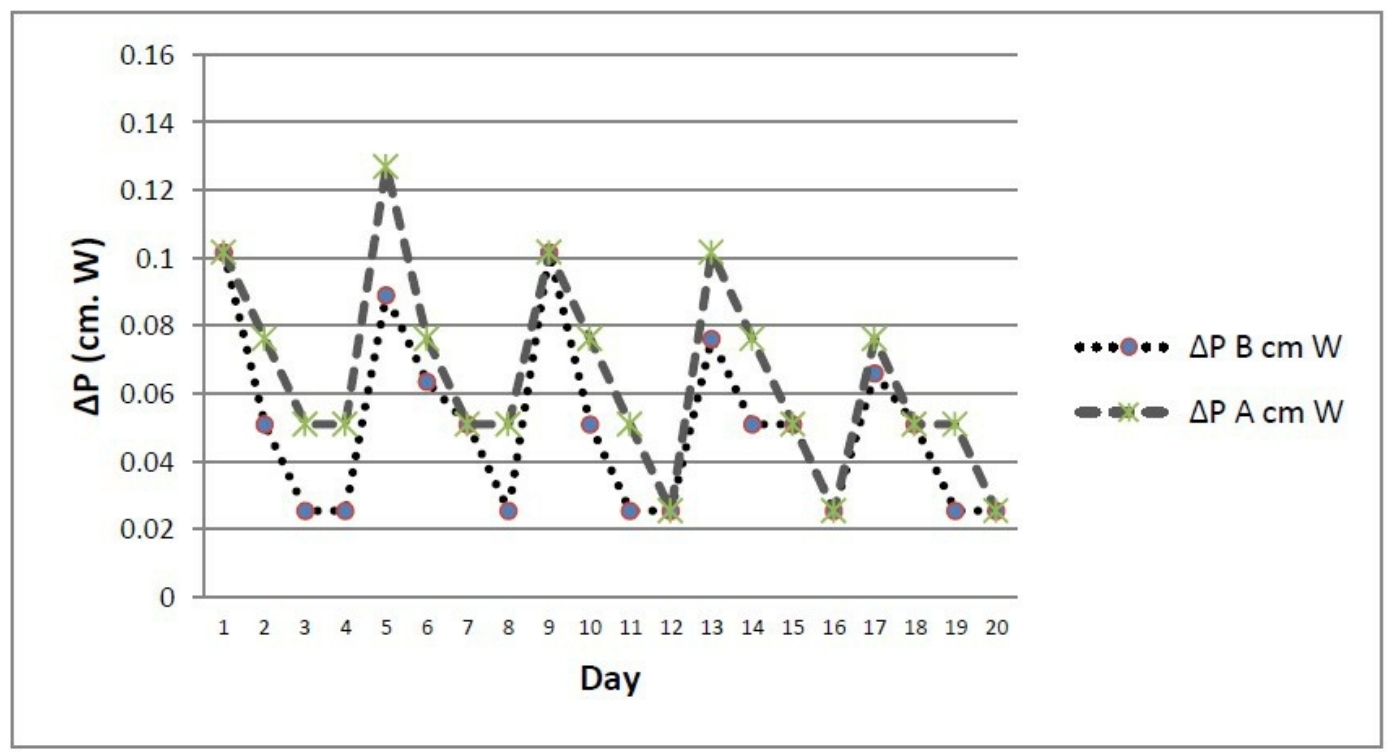

Figure 4

pressure drop as a function of time for the two parallel biofilters (BF-A, BF-B)

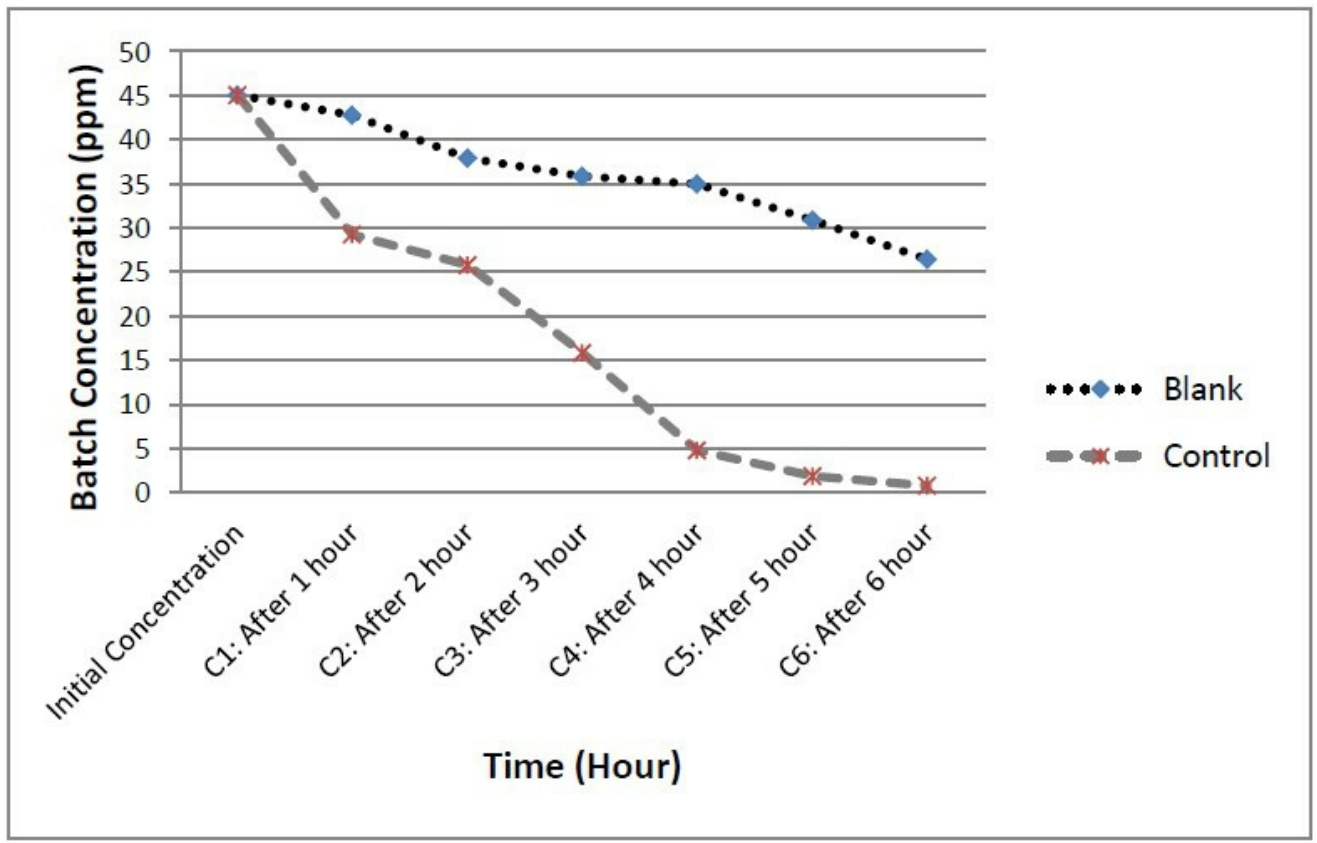

Figure 5

Biodegradation of toluene by pseudomonas putida, control $(\times)$ and blank ( $)$ for 45 ppmv 


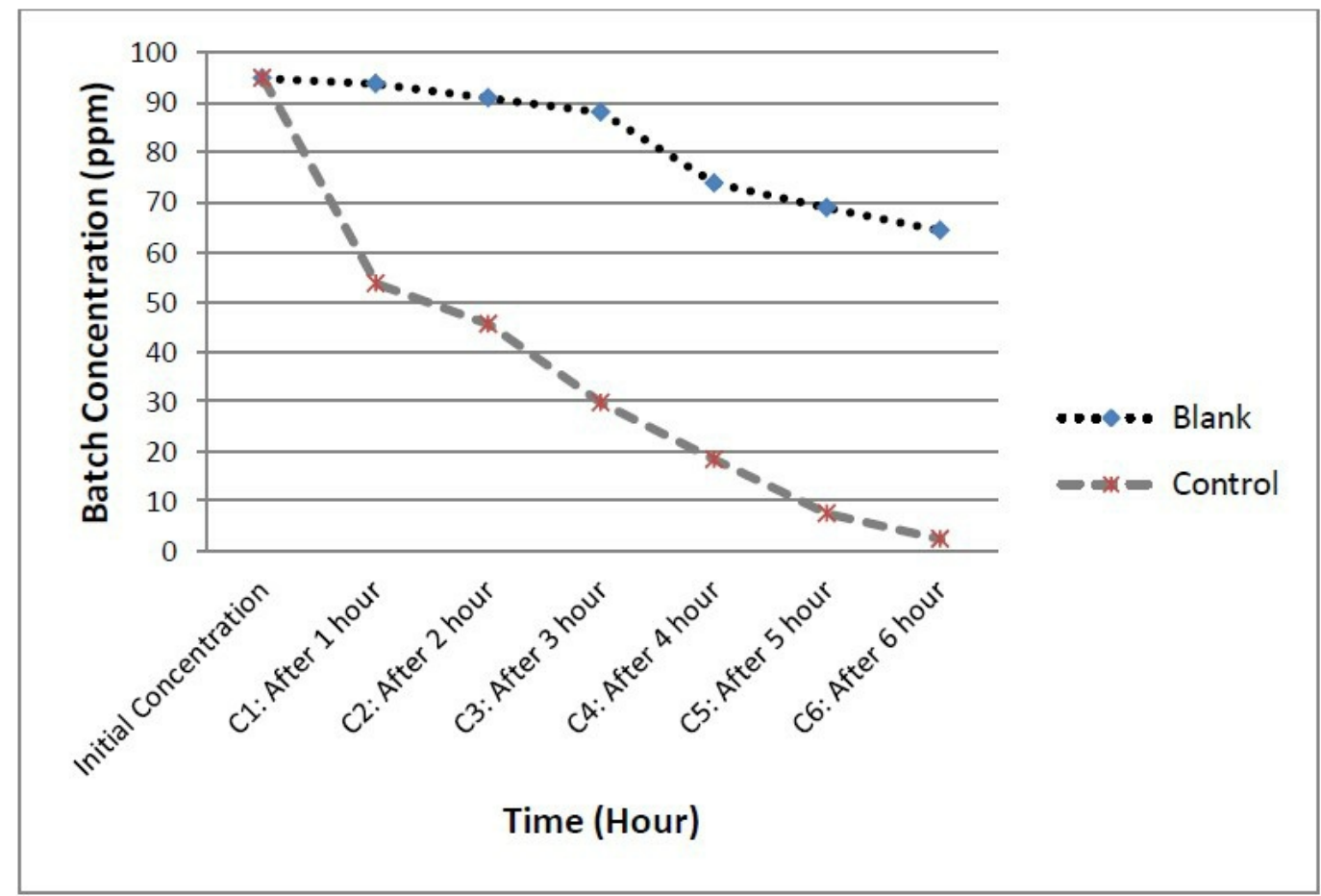

Figure 6

Biodegradation of toluene by pseudomonas putida, control $(x)$ and blank ( $\downarrow)$ for 95 ppmv 


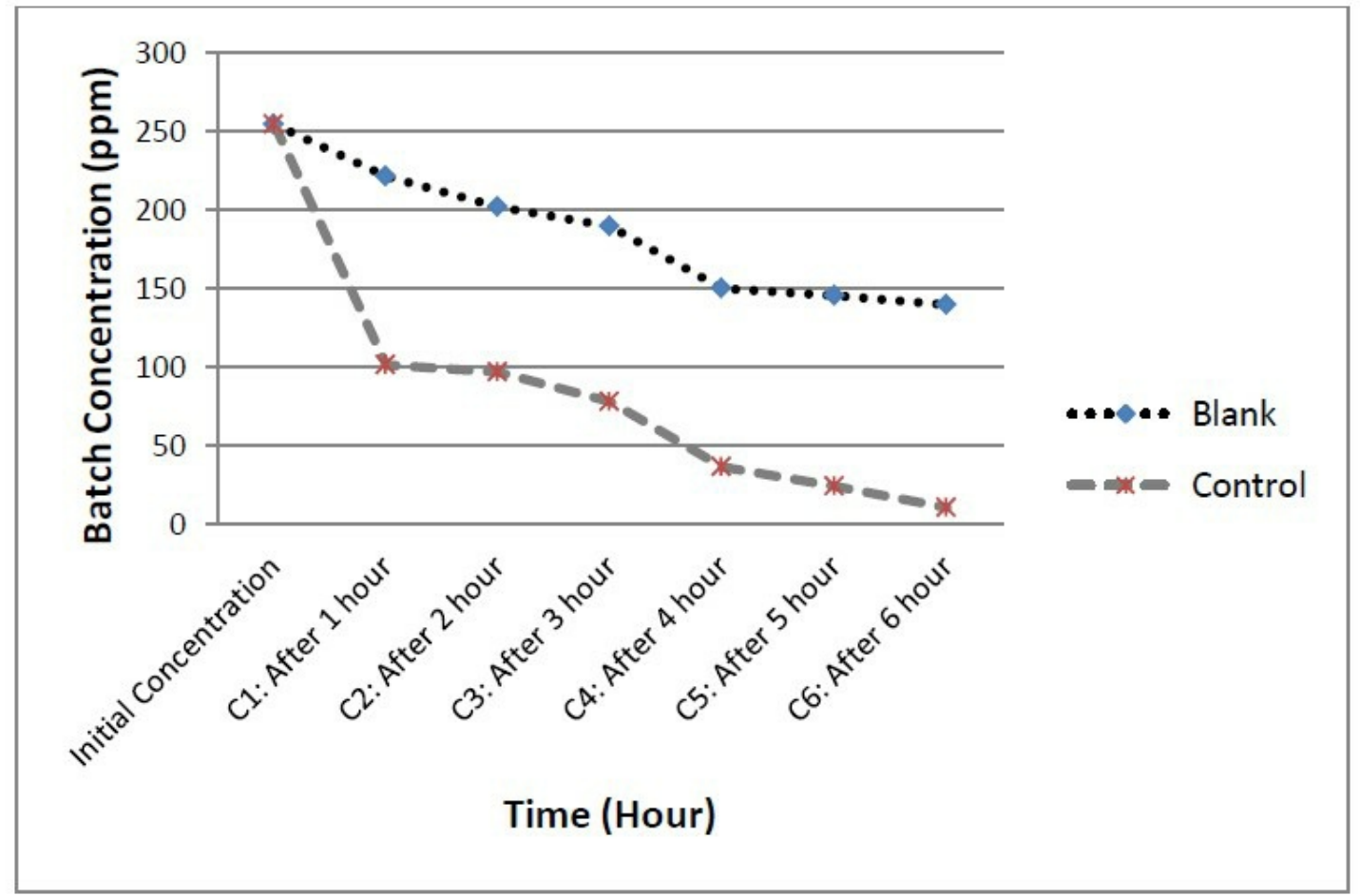

Figure 7

Biodegradation of toluene by pseudomonas putida, control $(x)$ and blank ( $)$ for 255 ppmv 